\title{
Brief Report: Visual-Spatial Deficit in a 16-year-old Girl with Maternally derived Duplication of Proximal 15q
}

David Cohen · Claire Martel · Anna Wilson •

Nicole Déchambre · Céline Amy · Ludovic Duverger · Jean-Marc Guile $\cdot$ Eva Pipiras · Brigitte Benzacken · Hélène Cavé · Laurent Cohen · Delphine Héron · Monique Plaza

Published online: 8 December 2006

(C) Springer Science+Business Media, LLC 2006

\section{Erratum to: J Autism Dev Disord}

\section{DOI 10.1007/s10803-006-0228-5}

In Table 1, please read as follows patient's mathematical abilities:

\section{Table 1}

\begin{tabular}{ll}
\hline Logical categorical thinking UDN-II & Acquired ( $>11$ years) \\
Spatial seriation UDN-II & Not acquired (<7 years) \\
Numerical seriation UDN-II & Not acquired (<10 years) \\
Inclusion UDN-II & Acquired ( $>11$ years) \\
Geometry REY FIGURE & $18 / 36(<-2 S D)$ \\
\hline
\end{tabular}

The online version of the original article can be found at http:// www.dx.doi.org/10.1007/s10803-006-0228-5

D. Cohen $(\bowtie) \cdot$ C. Martel · N. Déchambre .

C. Amy · L. Duverger

Département de Psychiatrie de l'Enfant et de l'Adolescent,

Université Pierre et Marie Curie, Groupe Hospitalier Pitié-

Salpétrière, AP-HP, 47 bd de l'Hôpital, 75013 Paris, France

e-mail: david.cohen@psl.aphp.fr

D. Cohen - J.-M. Guile $\cdot$ M. Plaza

CNRS, "Cognition et comportement", Université Paris V, Boulogne, France

A. Wilson - L. Cohen

INSERM-CEA Unit 562 "Cognitive Neuroimaging",

Service Hospitalier Frédéric Joliot, CEA-DRM-DSV,

Orsay, France
J.-M. Guile

Département de Psychiatrie, Université de Montréal,

Montréal, Canada

E. Pipiras · B. Benzacken

Service de Cytogénétique, Groupe Hospitalier Jean

Verdier, Bondy, France

H. Cavé

Service de Biochimie génétique, Groupe Hospitalier Robert Debré, Paris, France

D. Héron

Fédération de Génétique, Groupe Hospitalier PitiéSalpétrière, Paris, France 